\title{
D mesons in strongly magnetized asymmetric nuclear matter
}

\author{
Sushruth Reddy P* Amal Jahan CS \pm N Nikhil Dhale. \pm and Amruta Mishra $\$$ \\ Department of Physics, Indian Institute of Technology, \\ Delhi, Hauz Khas, New Delhi - 110 016, India \\ Juergen Schaffner-Bielich \\ Institut fuer Theoretische Physik, Goethe Universitaet, Frankfurt, \\ Max-von-Laue Str. 1, D-60438,Frankfurt am Main, Germany
}

\begin{abstract}
The medium modifications of the open charm mesons ( $D$ and $\bar{D})$ are studied in isospin asymmetric nuclear matter in the presence of strong magnetic fields, using a chiral effective model. The mass modifications of these mesons in the effective hadronic model, arise due to their interactions with the protons, neutrons and the scalar mesons (non-strange isoscalar $\sigma$, strange isoscalar, $\zeta$ and non-strange isovector, $\delta$ ), in the magnetized nuclear matter. In the presence of magnetic field, for the charged baryon, i.e., the proton, the number density as well as the scalar density have contributions due to the summation over the Landau energy levels. For a given value of the baryon density, $\rho_{B}$, and isospin asymmetry, the scalar fields are solved self consistently from their coupled equations of motion. The modifications of the masses of the $D$ and $\bar{D}$ mesons are calculated from the medium modifications of the scalar fields and the nucleons. The effects of the anomalous magnetic moments of the nucleons on the masses of the open charm mesons are also investigated in the present work. The effects of isospin asymmetry as well as of the anomalous magnetic moments are observed to be prominent at high densities for large values of magnetic fields.
\end{abstract}

*Electronic address: sushruth.p7@gmail.com

${ }^{\dagger}$ Electronic address: amaljahan@gmail.com

${ }_{\ddagger}^{\ddagger}$ Electronic address: dhalenikhil07@gmail.com

$\S$ Electronic address: amruta@physics.iitd.ac.in

๑Electronic address: schaffner@astro.uni-frankfurt.de 


\section{INTRODUCTION}

The study of the medium modifications of the properties of hadrons is an important and challenging area of research in the strong interaction physics. The topic has attracted a lot of attention in the recent past, due to its relevance to the ultra-relavitistic heavy ion collision experiments. The medium modifications of the hadrons in the matter at high densities and/or temperatures resulting from these high energy nuclear collisions, affect the observables in these experiments. The effects of isospin asymmetry are also important to investigate, as the heavy ion collision experiments involve nuclei which have large isospin asymmetry, with the number of neutrons being much larger than the number of protons. It is also important to study the effects of magnetic fields on the properties of the hadrons in hot and/or dense matter, since huge magnetic fields are believed to be created in the noncentral ultra-relativistic heavy ion collision experiments. The magnetic fields created in the heavy ion experiments are estimated to be order of $e B \sim 2 m_{\pi}^{2}$ (corresponding to a magnetic field $\sim 6 \times 10^{18}$ Gauss) at RHIC, BNL, and, $e B \sim 15 m_{\pi}^{2}$ at LHC, CERN [1, 2]. Also, strong magnetic fields do exist in astrophysical compact objects, like magnetars, which may have magnetic fields of the order of $10^{15}-10^{16}$ Gauss [3] at the surface and the magnetic field could be more intense in the interior of these stars. The bulk matter existing inside the neutron star in the presence of magnetic field has been studied extensively in the literature [4 8]. At high densties, the neutron stars could have a quark core, comprising of electrically charge neutral strange quark matter in the presence of magnetic field [9, 10]. The study of strongly interacting matter in magnetic field has gained a lot of interest in the recent years, due to the novel effects like chiral magnetic effect [1] as well as inverse magnetic catalysis [11] exhibited in the presence of magnetic field.

The strong magnetic fields created in non-central ultrarelativistic heavy ion collisions decrease rapidly after the collision, as the ion remnants recede away from the collision zone. This leads to induced currents which slow down the decrease in the magnetic field [12 14]. The time evolution of the magnetic field depends crucially on the electrical conductivity of the medium, and the larger the electrical conductivity, the longer the magnetic field is sustained. The effects of the magnetic field in the medium also tend to increase the electrical conductivity [15] which slows down the decay of the magnetic field. Initially after the collision 
there is a fast decrease in the magnetic field, whereas at later times, the matter effects lead to slowing down the decay of the magnetic field [16]. The time evolution of magnetic field in the heavy ion collisions is still an open question. This requires proper estimate of the electrical conductivity of the medium as well as solutions of the magnetohydrodynamic (MHD) equations, which need further investigations.

The open charm mesons $(D$ and $\bar{D})$ mesons, have appreciable modifications in the hadronic medium, because, due to the presence of the light quark (antiquark). These mesons interact with the light quark condensates, which are modified significantly in the hadronic medium. On the other hand, the charmonium states have modifications due to interaction with the gluon condensates in the medium, which are observed to be have much smaller changes in the medium as compared to those of light quark condensates. The strong magnetic fields produced in the relativistic heavy ion collision experiments, e.g. at the RHIC, BNL and at LHC, CERN, has initiated investigations of the properties of these heavy flavour mesons, e.g., $D$ and $B$ mesons, [17-19], as well as charmonium states [20], in the presence of magnetic fields. The present work is a step in the direction of studying the masses of the $D$ and $\bar{D}$ mesons in the presence of strong magnetic fields using a chiral effective model.

The open heavy flavour mesons, e.g., $D$ and $\bar{D}$ mesons, have been studied in the literature, using the QCD sum rule approach [21, 22], the Quark Meson Coupling (QMC) model [23] (where the quarks within the hadrons interact via scalar and vector mesons [24]), the effective hadronic model, e.g., chiral SU(4) model [25-28] as well as using the coupled channel approach [29-32]. Due to the presence of a light quark, the scalar open charm (bottom) are observed to be modified appreciably in the nuclear medium [33], similar to the $D(B)$ meson. These heavy flavour mesons have been studied using QCD sum rule approach [33 37] as well as coupled channel approach [38]. In the present work, we use a chiral effective model, for the study of $D$ and $\bar{D}$ mesons in nuclear matter in presence of a magnetic field. The model based on chiral SU(3) symmetry, has been used extensively to study nuclear matter, finite nuclei [39], hyperonic matter [40], vector mesons [41], kaons and antikaons [42 45], as well as to study the charge neutral matter as the bulk matter comprising the (proto) neutron stars [46]. The model has been generalized to chiral SU(4), to derive the interactions of the charm mesons with the light hadronic sector, so as to investigate the mass modifications of the $D$ 
and $\bar{D}$ mesons [26 28], as well as of the strange charm mesons (the $D_{s}$ mesons) [47]. The model also incorporates the broken scale invariance of QCD [39, 40, 48] through a scalar dilaton field which mimicks the gluon condensates of QCD, within the effective hadronic model. The mass modifications of the heavy quarkonium mesons, e.g., charmonium [27, 28] and bottomonium states [49], are due to their interactions with the gluon condensate of QCD, and, within the effective chiral model, these arise due to the modifications of the dilaton field in the hadronic medium. The medium modifications of the partial decay widths of the charmonium states to $D \bar{D}$ have been studied in the literature as arising from the medium modifications of the $D$ and $\bar{D}$ meson masses [50], using a light quark pair creation model, namely ${ }^{3} P_{0}$ model [51, 52]. Using the ${ }^{3} P_{0}$ model, these decay widths in hadronic matter have also been studied, as arising due to the mass modifications of the open charm mesons and the charmonium states, calculated within the effective chiral model [28]. The in-medium charmonium decay widths have later been studied, using a field theoretic model of composite hadrons [53]. Generalizing the model to the bottom sector, the in-medium masses of the $B, \bar{B}$ mesons [54] as well as the strange bottom meson, $B_{s}$ mesons [55], as arising from their interactions to the baryons and the scalar mesons, have been studied. The open charm and open bottom mesons have been studied in the literature using pion exchange in an effective hadronic model [56] and the attractive interactions of the $\bar{D}$ and $B$ mesons in nuclear matter predict possibility of the bound states of these mesons with the nuclei [23]. Due to attractive interaction of $J / \psi$ in nuclear matter [27, 28, 57, 58], the bound states of the $J / \psi$ to nuclei have also been predicted within the QMC model [59]. Using the medium modifications of the masses of the open bottom mesons, $B$ and $\bar{B}$, as well as the bottomonium states, as calculated within the effective hadronic model, the partial decay widths of the $\Upsilon$-states to $B \bar{B}$, in hadronic matter have been studied using the field theoretic model of composite hadrons with quark constituents [60].

As has already been mentioned, the medium modifications of the masses of the $D$ and $\bar{D}$ mesons, arise due to their interactions with the baryons and the scalar mesons in isospin (a)symmetric hadronic matter. The scalar mesons as calculated in the chiral model in the strange hadronic matter are related to the light quark condensates, i.e., the non-strange $\langle\bar{q} q\rangle, q=u, d$, as well as the strange condensate $\langle\bar{s} s\rangle$ in the medium. From the medium 
modifications of the scalar mesons, which are related to the quark condensates of QCD, the in-medium masses of the light vector mesons, namely, the $\omega, \rho$ and $\phi$ mesons [61], as well as the charmonium states, $J / \psi$ and $\eta_{c}$ [62] have been studied, using a QCD sum rule approach. The $D$ and $B$ mesons in the presence of an external magnetic field have been studied in the literature using a semiclassical approach [17], where the mass of the open charm (bottom) meson is due to the interaction of the magnetic field to the spin of the quarks. The magnetic field was observed to lead to different masses for the different spin orientations of the heavy-light quark-antiquark systems. In the presence of strong magnetic fields, there is mixing of the spin 0 states $D(B)$ to the spin 1 states $D^{*}\left(B^{*}\right)$. The D and $\mathrm{B}$ mesons were observed to have lowering of their masses (arising from the mass reductions of specific spin orientations) [17]. The effects of these mass reductions on the production of the charmonium and bottomonium states were studied using the color evaporation model in Ref. [17]. The $D$ mesons in the presence of a magnetic field have also been studied within the QCD sum rule approach, accounting for the mixing of the pesudoscalar mesons and the vector mesons, as well as the Landau quantization effects for the charged $D$ mesons [19]. This showed an increase in the mass of the neutral $D$ mesons, whereas, the mass spectra of the charged $D$ mesons turned out to saturate, in the presence of mixing as well as Landau quantization effects. The mass of charged $B$ mesons within the QCD sum rule approach were observed to decrease in the presence of magnetic fields [18]. The mass modifications of the charmonium ground states $\left(\eta_{c}\right.$ and $\left.J / \psi\right)$ have also been studied using the QCD sum rule approach [20] accounting for the effects of the mixing between these pesudoscalar and vector mesons. In the present work, the effects of the magnetic fields are considered to study the masses of the $D$ and $\bar{D}$ mesons in isospin asymmetric nuclear matter, using the chiral effective model. The mass modifications of the $D$ and $\bar{D}$ mesons arise due to their interactions with the nucleons and the scalar mesons in the magnetized hadronic matter. For given values of baryon density, $\rho_{B}$, and isospin asymmetry parameter, $\eta=\left(\rho_{n}-\rho_{p}\right) /\left(2 \rho_{B}\right)$, in the mean field approximation, the values of the scalar meson fields, $\sigma(\sim(u \bar{u}+d \bar{d})), \zeta(\sim s \bar{s})$ and $\delta(\sim(u \bar{u}-d \bar{d}))$, are calculated self-consistently from their equations of motion. There are contributions from the Landau energy levels for the number density as well as the scalar density, for the proton, the charged baryon, in the presence of the external magnetic field. 
The $D\left(D^{0}, D^{+}\right)$and $\bar{D}\left(\bar{D}^{0}, D^{-}\right)$meson in-medium masses, due to their interactions with the nucleons as well as the scalar mesons, are studied in the present work. The charged $D$ mesons ( $D^{ \pm}$mesons), additionally have a positive shift in their masses, due to Landau quantization, in the presence of the external magnetic field. The effects of anomalous magnetic moments of the proton and neutron [5-86, 63 66] are also investigated and these contributions on the $D$ meson masses are compared to the case when these effects are not taken into account. The effects of magnetic fields are observed to be large at high densities. At large magnetic fields, the effects of the anomalous magnetic moments are seen to be significant at high densities. The effects of isospin asymmetry is observed to be large at high densities, when the magnetic field is increased. In the present work, the effects on the masses of the $D$ and $\bar{D}$ mesons due to the mixing of the pesudoscalar and vector mesons [17 19] in the presence of magnetic fields, have not been taken into consideration, as these effects are not within the scope of the mean field approximation used in the chiral effective model. Moreover, the effects of temperature on the masses of the $D$ and $\bar{D}$ mesons were observed to be marginal as compared to the effects of the density, within the chiral effective model [27]. As a first step calculation, in the present work, the mass modifications of the $D$ and $\bar{D}$ mesons in asymmetric nuclear matter have been studied in the presence of strong magnetic fields, without accounting for the effects from temperature.

We organize the paper as follows: We briefly recapitulate the $S U(3)$-flavor chiral model adopted for the description of the asymmetric hadronic matter [44, 45] in the presence of an external magnetic field, in Section II. The number density as well as the scalar density of the charged baryon, i.e. the proton, have contributions from the Landau energy levels. We have additionally taken into account the effects of the anomalous magnetic moments of the proton and neutron for the study of the properties of the hadrons. The medium modifications of the $D$ and $\bar{D}$ meson masses are calculated through their interactions with the nucleons and scalar mesons, as has been described in Section III. Section IV discusses the results of the present investigation, while we summarise our findings and discuss possible outlook in Section V. 


\section{THE HADRONIC CHIRAL $S U(3) \times S U(3)$ MODEL}

The effective hadronic chiral Lagrangian density in the presence of magnetic field, used in the present work is given as

$$
\mathcal{L}=\mathcal{L}_{\text {kin }}+\sum_{W=X, Y, V, \mathcal{A}, u} \mathcal{L}_{B W}+\mathcal{L}_{\text {vec }}+\mathcal{L}_{0}+\mathcal{L}_{\text {scalebreak }}+\mathcal{L}_{S B}+\mathcal{L}_{\text {mag }}
$$

The above Lagrangian density is based on a nonlinear realization of SU(3) chiral symmetry [67 69] and broken scale invariance [39, 40, 48]. In Eq. (11), $\mathcal{L}_{\text {kin }}$ is the kinetic energy term, $\mathcal{L}_{B W}$ contains the baryon-meson interactions in which the baryon-scalar meson interaction terms generate the baryon masses. $\mathcal{L}_{\text {vec }}$ describes the dynamical mass generation of the vector mesons via couplings to the scalar fields and contains additionally quartic selfinteractions of the vector fields. $\mathcal{L}_{0}$ contains the meson-meson interaction terms inducing the spontaneous breaking of chiral symmetry, $\mathcal{L}_{\text {scalebreak }}$ is a scale invariance breaking logarithmic potential, $\mathcal{L}_{S B}$ describes the explicit chiral symmetry breaking. The last term, $\mathcal{L}_{\text {mag }}$ is the contribution from the magnetic field, given as

$$
\mathcal{L}_{m a g}=-\bar{\psi}_{i} q_{i} \gamma_{\mu} A^{\mu} \psi_{i}-\frac{1}{4} \kappa_{i} \mu_{N} \bar{\psi}_{i} \sigma^{\mu \nu} F_{\mu \nu} \psi_{i}-\frac{1}{4} F^{\mu \nu} F_{\mu \nu}
$$

In the above, $\psi_{i}$ corresponds to the $i$-th baryon. The second term in equation (2) corresponds to the tensorial interaction with the electromagnetic field and is related to the anomalous magnetic moments of the baryons (proton and neutron, in the present investigation). In this term, $\mu_{N}$ is the Nuclear Bohr magneton, given as $\mu_{N}=e /\left(2 m_{N}\right)$, where $m_{N}$ is the vacuum mass of the nucleon. We choose the magnetic field to be uniform and along the z-axis, and take the vector potential to be $A^{\mu}=(0,0, B x, 0)$.

To investigate the hadronic properties in the medium, we write the Lagrangian density within the chiral SU(3) model in the mean field approximation and determine the expectation values of the meson fields by minimizing the thermodynamical potential [40, 41]. In the present work, we shall be using the frozen glueball approximation, i.e., fix $\chi=\chi_{0}$, the vacuum value of the dilaton field, $\chi$. This is due to the reason that the medium modification of the dilaton field is observed to be negligible as compared to the medium changes of the scalar fields, $\sigma, \zeta$ and $\delta$, and hence has marginal contribution to the medium modifications of the $D$ and $\bar{D}$ meson masses. The non-strange scalar field $\sigma$, strange scalar field $\zeta$ and 
scalar-isovector field $\delta$, are solved from the equations of motion, derived from the Lagrangian density. These coupled equations of motion are given as

$$
\begin{aligned}
& k_{0} \chi^{2} \sigma-4 k_{1}\left(\sigma^{2}+\zeta^{2}+\delta^{2}\right) \sigma-2 k_{2}\left(\sigma^{3}+3 \sigma \delta^{2}\right)-2 k_{3} \chi \sigma \zeta \\
- & \frac{d}{3} \chi^{4}\left(\frac{2 \sigma}{\sigma^{2}-\delta^{2}}\right)+\left(\frac{\chi}{\chi_{0}}\right)^{2} m_{\pi}^{2} f_{\pi}-\sum_{i} g_{\sigma i} \rho_{i}^{s}=0 \\
& k_{0} \chi^{2} \zeta-4 k_{1}\left(\sigma^{2}+\zeta^{2}+\delta^{2}\right) \zeta-4 k_{2} \zeta^{3}-k_{3} \chi\left(\sigma^{2}-\delta^{2}\right) \\
- & \frac{d}{3} \frac{\chi^{4}}{\zeta}+\left(\frac{\chi}{\chi_{0}}\right)^{2}\left[\sqrt{2} m_{k}^{2} f_{k}-\frac{1}{\sqrt{2}} m_{\pi}^{2} f_{\pi}\right]-\sum_{i} g_{\zeta i} \rho_{i}^{s}=0 \\
& k_{0} \chi^{2} \delta-4 k_{1}\left(\sigma^{2}+\zeta^{2}+\delta^{2}\right) \delta-2 k_{2}\left(\delta^{3}+3 \sigma^{2} \delta\right)+k_{3} \chi \delta \zeta \\
+ & \frac{2}{3} d\left(\frac{\delta}{\sigma^{2}-\delta^{2}}\right)-\sum_{i} g_{\delta i} \rho_{i}^{s}=0
\end{aligned}
$$

In the above, $\rho_{i}^{s}, i=p, n$, are the scalar densities for the proton and neutron respectively, in the magnetic field, $B$ chosen to be along the z-direction. For proton, the number density as well as the scalar density have contributions from the Landau energy levels in the presence of the magnetic field. The number density and the scalar density of the proton are given as [7, 8]

$$
\rho_{p}=\frac{e B}{4 \pi^{2}}\left[\sum_{\nu=0}^{\nu_{\max }^{(S=1)}} k_{f, \nu, 1}^{(p)}+\sum_{\nu=1}^{\nu_{(\max )}^{(S=-1)}} k_{f, \nu,-1}^{(p)}\right]
$$

and

$$
\begin{aligned}
\rho_{p}^{s} & =\frac{e B m_{p}^{*}}{2 \pi^{2}}\left[\sum_{\nu=0}^{\nu_{\text {max }}^{(S=1)}} \frac{\sqrt{m_{p}^{* 2}+2 e B \nu}+\Delta_{p}}{\sqrt{m_{p}^{* 2}+2 e B \nu}} \ln \left|\frac{k_{f, \nu, 1}^{(p)}+E_{f}^{(p)}}{\sqrt{m_{p}^{* 2}+2 e B \nu}+\Delta_{p}}\right|\right. \\
& \left.+\sum_{\nu=1}^{\nu_{\max }^{(S=-1)}} \frac{\sqrt{m_{p}^{* 2}+2 e B \nu}-\Delta_{p}}{\sqrt{m_{p}^{* 2}+2 e B \nu}} \ln \left|\frac{k_{f, \nu,-1}^{(p)}+E_{f}^{(p)}}{\sqrt{m_{p}^{* 2}+2 e B \nu}-\Delta_{p}}\right|\right]
\end{aligned}
$$

where, $k_{f, \nu, \pm 1}^{(p)}$ are the Fermi momenta of protons for the Landau level, $\nu$ for the spin index, $S= \pm 1$, i.e. for spin up and spin down projections for the proton. These Fermi momenta are related to the Fermi energy of the proton as

$$
k_{f, \nu, S}^{(p)}=\sqrt{E_{f}^{(p)^{2}}-\left(\sqrt{m_{p}^{* 2}+2 e B \nu}+S \Delta_{p}\right)^{2}} .
$$


The number density and the scalar density of neutrons are given as

$$
\rho_{n}=\frac{1}{4 \pi^{2}} \sum_{S= \pm 1}\left\{\frac{2}{3} k_{f, S}^{(n)^{3}}+S \Delta_{n}\left[\left(m_{n}^{*}+S \Delta_{n}\right) k_{f, S}^{(n)}+E_{f}^{(n)^{2}}\left(\arcsin \left(\frac{m_{n}^{*}+S \Delta_{n}}{E_{f}^{(n)}}\right)-\frac{\pi}{2}\right)\right]\right\}
$$

and

$$
\rho_{n}^{s}=\frac{m_{n}^{*}}{4 \pi^{2}} \sum_{S= \pm 1}\left[k_{f, S}^{(n)} E_{f}^{(n)}-\left(m_{n}^{*}+S \Delta_{n}\right)^{2} \ln \left|\frac{k_{f, S}^{(n)}+E_{f}^{(n)}}{m_{n}^{*}+S \Delta_{n}}\right|\right] .
$$

The Fermi momentum, $k_{f, S}^{(n)}$ for the neutron with spin projection, $\mathrm{S}(S= \pm 1$ for the up (down) spin projection), is related to the Fermi energy for the neutron, $E_{f}^{(n)}$ as

$$
k_{f, S}^{(n)}=\sqrt{E_{f}^{(n)^{2}}-\left(m_{n}^{*}+S \Delta_{n}\right)^{2}} .
$$

In the above, the parameter $\Delta_{i}$ refers to the anomalous magnetic moment for the baryon, $i$ $(i=p, n)$, given as

$$
\Delta_{i}=-\frac{1}{2} \kappa_{i} \mu_{N} B
$$

where, $\kappa_{i}$, is as defined in the electromagnetic tensor term in the Lagrangian density given by (2). The values of $\kappa_{p}$ and $\kappa_{n}$ are given as 3.5856 and -3.8263 respectively, which are the values of the gyromagnetic ratio corresponding to the anomalous magnetic moments of the proton and neutron respectively.

In the following section, we shall study the medium modifications of the $D$ and $\bar{D}$ mesons in the isopsin asymmetric nuclear matter in the presence of a magnetic field. We shall consider the effects of the anomalous magnetic moments of the baryons, on the mass modifications of these mesons, and compare the results for the $D$ and $\bar{D}$ meson masses, when these effects are not taken into account.

\section{IN MEDIUM MASSES OF $D$ AND $\bar{D}$ MESONS IN MAGNETIZED ISOSPIN ASYMMETRIC NUCLEAR MATTER}

The in-medium changes of the $D$ and $\bar{D}$ mesons in asymmetric nuclear matter are studied in the presence of strong external magnetic fields. These medium modifications are investigated using a chiral effective model, where the chiral SU(3) has been generalized to chiral $\mathrm{SU}(4)$ in order to derive the interactions of the charmed mesons with the light hadronic sector [25-28]. 
The interaction Lagrangian modifying the $D$-meson mass can be written as [44]

$$
\begin{aligned}
\mathcal{L}_{\mathcal{D N}} & =-\frac{i}{8 f_{D}^{2}}\left[3\left(\bar{p} \gamma^{\mu} p+\bar{n} \gamma^{\mu} n\right)\left(D^{0}\left(\partial_{\mu} \bar{D}^{0}\right)-\left(\partial_{\mu} D^{0}\right) \bar{D}^{0}\right)+\left(D^{+}\left(\partial_{\mu} D^{-}\right)-\left(\partial_{\mu} D^{+}\right) D^{-}\right)\right. \\
& \left.+\left(\bar{p} \gamma^{\mu} p-\bar{n} \gamma^{\mu} n\right)\left(D^{0}\left(\partial_{\mu} \bar{D}^{0}\right)-\left(\partial_{\mu} D^{0}\right) \bar{D}^{0}\right)-\left(D^{+}\left(\partial_{\mu} D^{-}\right)-\left(\partial_{\mu} D^{+}\right) D^{-}\right)\right] \\
& \left.+\frac{m_{D}^{2}}{2 f_{D}}\left[\left(\sigma+\sqrt{2} \zeta_{c}\right)\left(\bar{D}^{0} D^{0}+\left(D^{-} D^{+}\right)\right)+\delta\left(\bar{D}^{0} D^{0}\right)-\left(D^{-} D^{+}\right)\right)\right] \\
& -\frac{1}{f_{D}}\left[\left(\sigma+\sqrt{2} \zeta_{c}\right)\left(\left(\partial_{\mu} \bar{D}^{0}\right)\left(\partial^{\mu} D^{0}\right)+\left(\partial_{\mu} D^{-}\right)\left(\partial^{\mu} D^{+}\right)\right)\right. \\
& \left.+\delta\left(\left(\partial_{\mu} \bar{D}^{0}\right)\left(\partial^{\mu} D^{0}\right)-\left(\partial_{\mu} D^{-}\right)\left(\partial^{\mu} D^{+}\right)\right)\right] \\
& +\frac{d_{1}}{2 f_{D}^{2}}(\bar{p} p+\bar{n} n)\left(\left(\partial_{\mu} D^{-}\right)\left(\partial^{\mu} D^{+}\right)+\left(\partial_{\mu} \bar{D}^{0}\right)\left(\partial^{\mu} D^{0}\right)\right) \\
& +\frac{d_{2}}{4 f_{D}^{2}}[(\bar{p} p+\bar{n} n))\left(\left(\partial_{\mu} \bar{D}^{0}\right)\left(\partial^{\mu} D^{0}\right)+\left(\partial_{\mu} D^{-}\right)\left(\partial^{\mu} D^{+}\right)\right) \\
& \left.\left.+(\bar{p} p-\bar{n} n)\left(\left(\partial_{\mu} \bar{D}^{0}\right)\left(\partial^{\mu} D^{0}\right)\right)-\left(\partial_{\mu} D^{-}\right)\left(\partial^{\mu} D^{+}\right)\right)\right]
\end{aligned}
$$

In (13), the first term is the vectorial Weinberg Tomozawa interaction term, which is attractive for $D$ mesons, but repulsive for the $\bar{D}$ mesons. The second term is the scalar meson exchange term, which is attractive for both $D$ and $\bar{D}$ mesons. The third, fourth and fifth terms comprise the range term in the chiral model. The parameters $d_{1}$ and $d_{2}$ in the last two terms of the interaction Lagrangian given by (13) are determined by fitting to the empirical values of the KN scattering lengths [70 72] for $I=0$ and $I=1$ channels [44, 45].

The dispersion relations for the $D$ and $\bar{D}$ mesons are obtained from the Fourier transformations of the equations of motion of these mesons. These are given as

$$
-\omega^{2}+\vec{k}^{2}+m_{D(\bar{D})}^{2}-\Pi_{D(\bar{D})}(\omega,|\vec{k}|)=0
$$

where $\Pi_{D(\bar{D})}$ denotes the self energy of the $D(\bar{D})$ meson in the medium. For the $D$ meson doublet $\left(D^{0}, D^{+}\right)$, and $\bar{D}$ meson doublet $\left(\bar{D}^{0}, D^{-}\right)$, the self enrgies are given by

$$
\begin{aligned}
\Pi(\omega,|\vec{k}|) & \left.=\frac{1}{4 f_{D}^{2}}\left[3\left(\rho_{p}+\rho_{n}\right) \pm\left(\rho_{p}-\rho_{n}\right)\right)\right] \omega \\
& +\frac{m_{D}^{2}}{2 f_{D}}\left(\sigma^{\prime}+\sqrt{2} \zeta_{c}{ }^{\prime} \pm \delta^{\prime}\right) \\
& +\left[-\frac{1}{f_{D}}\left(\sigma^{\prime}+\sqrt{2} \zeta_{c}{ }^{\prime} \pm \delta^{\prime}\right)+\frac{d_{1}}{2 f_{D}^{2}}\left(\rho_{p}^{s}+\rho_{n}^{s}\right)\right. \\
& \left.+\frac{d_{2}}{4 f_{D}^{2}}\left(\left(\rho_{p}^{s}+\rho_{n}^{s}\right) \pm\left(\rho_{p}^{s}-\rho_{n}^{s}\right)\right)\right]\left(\omega^{2}-\vec{k}^{2}\right)
\end{aligned}
$$


and

$$
\begin{aligned}
\Pi(\omega,|\vec{k}|) & =-\frac{1}{4 f_{D}^{2}}\left[3\left(\rho_{p}+\rho_{n}\right) \pm\left(\rho_{p}-\rho_{n}\right)\right] \omega \\
& +\frac{m_{D}^{2}}{2 f_{D}}\left(\sigma^{\prime}+\sqrt{2} \zeta_{c}{ }^{\prime} \pm \delta^{\prime}\right) \\
& +\left[-\frac{1}{f_{D}}\left(\sigma^{\prime}+\sqrt{2} \zeta_{c}^{\prime} \pm \delta^{\prime}\right)+\frac{d_{1}}{2 f_{D}^{2}}\left(\rho_{p}^{s}+\rho_{n}^{s}\right)\right. \\
& +\frac{d_{2}}{4 f_{D}^{2}}\left(\left(\rho_{p}^{s}+\rho_{n}^{s}\right) \pm\left(\rho_{n}^{s}-\rho_{n}^{s}\right)\right]\left(\omega^{2}-\vec{k}^{2}\right)
\end{aligned}
$$

where the \pm signs refer to the $D^{0}$ and $D^{+}$respectively in equation (15) and to the $\bar{D}^{0}$ and $D^{-}$respectively in equation (16). In equations (15) and (16), $\sigma^{\prime}\left(=\left(\sigma-\sigma_{0}\right)\right), \zeta_{c}^{\prime}(=$ $\left.\left(\zeta_{c}-\zeta_{c 0}\right)\right)$ and $\delta^{\prime}\left(=\left(\delta-\delta_{0}\right)\right)$ are the fluctuations of the scalar-isoscalar fields $\sigma$ and $\zeta_{c}$, and the third component of the scalar-isovector field, $\delta$, from their vacuum expectation values. The vacuum expectation value of $\delta$ is zero $\left(\delta_{0}=0\right)$, since a nonzero value for it will break the isospin symmetry of the vacuum. In the above, $\rho_{p}$ and $\rho_{n}$ are the number densities of proton and neutron and $\rho_{p}^{s}$ and $\rho_{n}^{s}$ are their scalar densities.

The masses of the charged open charm mesons, $D^{ \pm}$have an additional positive mass shift due to the presence of the magnetic field, which retaining only the lowest Landau level, is given as

$$
m_{D^{ \pm}}^{e f f}=\sqrt{m_{D^{ \pm}}^{*}+|e B|}
$$

whereas for the neutral $D(\bar{D})$ mesons, namely, $D^{0}\left(\bar{D}^{0}\right)$, the effective masses are given as

$$
m_{D^{0}\left(\overline{D^{0}}\right)}^{e f f}=m_{D^{0}\left(\overline{D^{0}}\right)}^{*},
$$

In the above, $m_{D(\bar{D})}^{*}$ are the solutions for $\omega$ at $|\vec{k}|=0$, of the dispersion relations given by equation (14).

In the next section, we shall discuss the results for the $D(\bar{D})$-meson mass modifications in (a)symmetric nuclear matter in the presence of an external magnetic field, as obtained in the present effective chiral model. 


\section{RESULTS AND DISCUSSIONS}

The in-medium masses of the $D$ and $\bar{D}$ mesons are investigated in asymmetric nuclear matter in the presence of an external magnetic field, using a chiral effective model. The medium modifications arise due to the interactions of these open charm mesons with the protons, neutrons and the scalar mesons $(\sigma, \zeta$ and $\delta)$. The number density and scalar density of the charged baryon, the proton, have contributions from the Landau energy levels, in the presence of the external magnetic field, $\vec{B}=(0,0, B)$. The in-medium masses of the $D$ and $\bar{D}$ mesons are calculated by using the dispersion relation given by (14), with the self-energies for the $D\left(D^{0}, D^{+}\right)$and $\bar{D}\left(\bar{D}^{0}, D^{-}\right)$mesons given by equations (15) and (16) respectively. The charged $D$ and $\bar{D}$ mesons, i.e., $D^{ \pm}$have additional mass modifications due to the Landau quantization effects in the presence of the external magnetic field, as given by equation (17).

The expectation values of the scalar fields, $\sigma, \zeta$ and $\delta$, are solved from the equations of motion of these mesons, given by equations (3), (4) and (15). The values of $\sigma, \zeta$ and $\delta$ are plotted as functions of $\rho_{B} / \rho_{0}$ (the baryon density in units of the nuclear matter saturation density) in figures 1, 2, and 3, respectively. These are shown for various values of the magnetic field, as well as for different values of the isospin asymmetry parameter, $\eta=\left(\rho_{n}-\rho_{p}\right) /\left(2 \rho_{B}\right)$, including the effects from the anomalous magnetic moments (AMM) of the nucleons. These are compared with the case of without accounting for the effects from the anomalous magnetic moments (shown as dotted lines). In figure 1, the isospin asymmetry effects on the scalar field $\sigma$ are illustrated for values of the magnetic field $e B$ as $2 m_{\pi}^{2}, 4 m_{\pi}^{2}, 6 m_{\pi}^{2}$, and $8 m_{\pi}^{2}$, in panels (a), (b), (c) and (d) respectively. For isospin symmetric nuclear matter, the effect of the anomalous magnetic moments is observed to give smaller values of the scalar densities of the proton and neutron, as compared to the case when these

effects are neglected. This leads to the magnitude of the $\sigma$ with AMM effects, to be larger, as can be inferred from the equation for $\sigma$ given by equation (3). There is observed to be an increase in the magnitude of the value of $\sigma$, when one goes from symmetric nuclear matter to asymmetric nuclear matter. This is a reflection of the fact that the value of $\left(\rho_{p}^{s}+\rho_{n}^{s}\right)$ is smaller for the isospin asymmetric case. The effects of the isospin asymetry as well as anomalous magnetic moments are seen to be larger at high densities. These effects are observed to be larger with increase in the magnetic field, as can be seen in figure 1. For the 
case of $\zeta$ meson, the behaviour of the expectation value of the field, is seen to be similar to that of the $\sigma$ meson. However, the change is observed to be much smaller than that of the $\sigma$ field.

In figure 3, the $\delta$ field is plotted as a function of the baryon density (in units of the nuclear matter saturation density, $\rho_{0}$ ), for different values of magnetic fields, and the effect of the isospin asymmetry on the value of $\delta$ is also investigated. For the isospin symmetric case $\left(\eta=0\right.$, i.e., $\left.\rho_{p}=\rho_{n}\right)$, the value of $\delta$ (which is determined by the difference of $\left(\rho_{p}^{s}-\rho_{s}^{n}\right)$ ), is zero for the case when there is no external magnetic field, as $\rho_{p}^{s}=\rho_{n}^{s}$ in this case. This is because the Fermi momentum for the proton is equal to that of the neutron for $\rho_{p}=\rho_{n}$, and hence $\rho_{p}^{s}=\rho_{n}^{s}$, for the case of zero magnetic field, which gives the value of $\delta$ to be zero for symmetric nuclear matter $(\eta=0)$ in the absence of magnetic field. However, in the presence of magnetic field, in symmetric nuclear matter, the proton, being the charged nucleon, has contributions from the Landau levels. The scalar density of proton, $\rho_{p}^{s}$ thus turns out to be different from the scalar density of neutron, $\rho_{n}^{s}$, and the value of $\delta$, as obtained from the solutions of the coupled equations for the scalar fields, given by equations (3), (4) and (15), is found to be nonzero and positive, as can be seen from figure 3. When the anomalous magnetic moments (AMM) of the nucleons are taken into account, for symmetric nuclear matter $(\eta=0)$ there is observed to be an intial increase in the value of $\delta$ with density reaching a maximum value, followed by a drop when the density is further increased. The value of $\delta$ is observed to increase with increase in the magnetic field, reaching a maximum value of around $0.6 \mathrm{MeV}$, for $e B=8 m_{\pi}^{2}$ at a density of around $3 \rho_{0}$, when the effects of anomalous magnetic moments (AMM) of the nucleons are taken into account. The values of $\delta$ for $\eta=0$ remain similar when the AMM effects are not taken into account. However, there is observed to be a monotonic increase in the value of $\delta$ with density in the latter case. For isospin asymmetry parameter, $\eta=0.3$, in the absence of AMM effects, there is observed to be an initial increase in the magnitude of the $\delta$ field upto a density of around $2 \rho_{0}$, followed by a drop in the magnitude when the density is further increased. The behaviour is related to the similar behaviour of $\left(\rho_{p}^{s}-\rho_{n}^{s}\right)$, with density, for this value of the isospin asymmetry parameter $(\eta=0.3)$. Inclusion of the effects of anomalous magnetic moments, leads to first a drop, i.e., $\delta$ becomes more negative, and then a slight increase in the value of $\delta$ with density, for $e B=2 m_{\pi}^{2}$, whereas 
for the higher values of the magnetic field, the value remains almost unchanged at densities higher than around $2.5 \rho_{0}$. For the maximum asymmetric case $(\eta=0.5)$, i.e., when there are only neutrons in the system, the anomalous magnetic moment due to the neutron is observed to give rise to a smaller magnitude for the $\delta$ field, for densities in the range of $\rho_{0}$ to $4 \rho_{0}$. For densities larger than $4 \rho_{0}$, the value of $\delta$ is observed to be very similar for both the cases of with and without accounting for the anomalous magnetic moment effects, for $\eta=0.5$.

The dependence of the scalar fields, $\sigma, \zeta$ and $\delta$ on the baryon density is observed to be the dominant medium effect as compared to the dependence of these fields on the magnetic field, as can be seen from figures 1, 2] and 3. In isospin symmetric matter, at $\rho_{B}=\rho_{0}$, the value of $\sigma$ changes only marginally with increase in magnetic field. For example, the values of $\sigma$ (in $\mathrm{MeV}$ ) at $e B$ as equal to $m_{\pi}^{2}, 5 m_{\pi}^{2}$ and $8 m_{\pi}^{2}$, are observed to be $-60.54(-60.31),-60.4(-59.94)$ and $-60.42(-59.9)$ respectively, with (without) AMM effects. For $\eta=0.3$, these values of $\sigma($ in $\mathrm{MeV})$ are modified to $-60.83(-60.71),-61.45(-60.61)$ and $-61.58(-60.62)$ respectively. As the density is increased to $5 \rho_{0}$, the value of $\sigma$ is observed to be $-29(-28.91),-29.835(-28.07)$ and $-31(-26.74)$ for symmetric nuclear matter with (without) accounting for the AMM effects, for the value of $e B$ as equal to $m_{\pi}^{2}, 5 m_{\pi}^{2}$ and $8 m_{\pi}^{2}$ respectively. The scalar field $\zeta$ follows the same trend as $\sigma$, but the changes in case of $\zeta$ are found to be extremely small even at higher densities and high magnetic fields. The value of $\delta$ at small densities and low magnetic field is close to zero in symmetric nuclear matter. With the increase in density the value of $\delta$ becomes more noticeable and it increases further at higher magnetic fields. For $\eta=0.5$, and in the absence of the AMM effects, the scalar fields, $\sigma, \zeta$ and $\delta$, do not respond to changes in magnetic field and remain constant for a particular density as there are only neutrons in the medium for $\eta=0.5$, and neutrons are not subjected to magnetic field effects, when the anomalous magnetic moment effects are not taken into consideration.

The in-medium masses of the $D$ mesons $\left(D^{+}\right.$and $\left.D^{0}\right)$ as well as $\bar{D}\left(D^{-}\right.$and $\left.\bar{D}^{0}\right)$ mesons in magnetized nuclear matter are calculated from the interactions of these mesons as given by equation (13) and, from the Landau quantization for the charged $D^{ \pm}$mesons. The dependence of the masses of the $D^{+}, D^{0}, D^{-}$and $\bar{D}^{0}$ on the magnetic field, are illustrated for baryon densities $\rho_{0}, 3 \rho_{0}$ and $5 \rho_{0}$, in figures 4 , 5 and 6 respectively. The effects of the 
isospin asymmetry on the masses of these open charm mesons are also investigated. The in-medium masses of the $D^{+}$and $D^{0}$ mesons are shown in panels (a) and (b) of these figures. The isospin symmetric part $\left(\sim\left(\rho_{p}+\rho_{n}\right)\right)$ of the first term of the interaction Lagrangian, namely the Weinberg-Tomozawa term gives a drop in the $D^{+}$as well as the $D^{0}$ meson masses in the hadronic medium, as can be seen from dispersion relation (14) and the self energy for the $D$ meson given by equation (15). With isospin asymmetry introduced in the medium, the $D^{+}$experiences a further drop in the mass, whereas $D^{0}$ experiences a positive contribution to the mass, from the second term of the Weinbrg-Tomozawa term, thereby giving a mass splitting of $D^{+}$and $D^{0}$ mesons in the asymmetric nuclear matter. For isospin symmetric nuclear matter $(\eta=0)$, as has already been mentioned, in the presence of magnetic field, the unequal values of the scalar densities for the proton and neutron (due to Landau quantization for the proton) leads to nonzero value of $\delta$. This gives rise to a splitting in the masses of the $D^{+}$and $D^{0}$ in the presence of magnetic field, arising from the scalar exchange as well as the range terms, even in symmetric nuclear matter. The contributions of all the terms are observed to show a large drop of the $D^{+}$mass in the asymmetric nuclear medium, with the drop being larger for higher asymmetry in the medium. The $D^{+}$meson has a further contribution in the presence of magnetic field, due to Landau quantization, which gives a positive shift in its mass, as given by equation (17). For the $D^{0}$ meson, the isospin asymmetry is observed to give rise to a smaller drop in the in-medium mass arising from its interaction with the nucleons and the scalar mesons. The effects of isospin asymmetry are seen to be quite prominent for $D^{0}$ meson as compared to $D^{+}$meson for higher values of the densities, $3 \rho_{0}$ and $5 \rho_{0}$, plotted in figures 5 and $[6$. The effects of anomalous magnetic moment are observed to give a smaller drop of the masses of the $D^{+}$and $D^{0}$ mesons, and the difference as compared to when these effects are not accounted for, is seen to be high at high densities.

For $D^{+}$mesons, considering AMM effects, the mass obtained from dispersion relation, which is further shifted because of Landau quantization effects as given by equation (17), shows a steady increase with rising magnetic fields for all densities and isospin conditions of the medium. Without AMM effects, the increase in the mass is observed to be much less as compared to when these effects are not taken into account, and this is more noticeable for 
higher densities. For $\eta=0.3$ and $\rho_{B}=\rho_{0}$, for the values of $e B$ as $m_{\pi}^{2}, 5 m_{\pi}^{2}, 8 m_{\pi}^{2}$, the mass of $D^{+}$is observed to be 1783.17 (1781.86), 1807.0 (1802.96) and 1829.2 (1825.4) respectively with (without) AMM effects. When the density is increased to $5 \rho_{0}$, the value of $D^{+}$mass (in $\mathrm{MeV}$ ) is seen to be 1421.94 (1421), 1456 (1440) and 1498.8 (1462.6) respectively.

The $D^{0}$ meson shows only a small modification in its mass with respect to change in magnetic field at nuclear matter density, as can be seen from figure 4. At higher densities, $3 \rho_{0}$ and $5 \rho_{0}$, plotted in figures 5 and 6, the $D^{0}$ mass is observed to increase with rise in magnetic fields for isospin asymmetric nuclear matter, when the AMM effects are taken into account. When AMM effects are not considered, the $D^{0}$ mass is observed to remain smaller as compared to when these effects are considered, and the difference in these masses is seen to reduce with increase in isospin asymmetry in the medium. In the absence of AMM effects, the $D^{0}$ mass remains constant in case of $\eta=0.5$, as the magnetic effects for neutrons are only due to their anomalous magnetic moments. For $\eta=0.3$ and $\rho_{B}=\rho_{0}$, the values of the $D^{0}$ mass (in $\mathrm{MeV}$ ) with (without) AMM effects, are found to be 1796.31 (1796.65) 1798.39 (1796.28) and 1798.7 (1795.8) for $e B$ as $m_{\pi}^{2}, 5 m_{\pi}^{2}, 8 m_{\pi}^{2}$ respectively. For $\rho_{B}=5 \rho_{0}$, these values of $D^{0}$ mass are modified to 1492.2 (1491.2) 1494.7 (1477.7) and 1506.3 (1472.1) respectively. For the symmetric as well as asymmetric (with $\eta=0.3$ ) nuclear matter, when the AMM effects are not included, the mass of the $D^{0}$ meson is observed to drop with increase in the magnetic field, and this behaviour is marginal for $\rho_{B}=\rho_{0}$, but quite appreciable for the higher densities of $3 \rho_{0}$ and $5 \rho_{0}$, as can be seen from the figures 4, 5 and 6 . The values of $D^{+}$as well as $D^{0}$ masses are unaffected by magnetic field for $\eta=0.5$, when AMM effects are not considered, as neutrons have only magnetic field effects from their AMMs.

The mass of the $D^{-}\left(\bar{D}^{0}\right)$ meson is observed to show similar behaviour when the magnetic field is increased, as the mass of the $D^{+}\left(D^{0}\right)$ meson, as can be seen from the figures 4, 5] and 6. plotted for densities $\rho_{0}, 3 \rho_{0}$ and $5 \rho_{0}$ respectively. For $\rho_{B}=\rho_{0}$, the mass modification of the $\bar{D}^{0}$ remains marginal with rise in the magnetic field, whereas for higher densities, the value of the $\bar{D}^{0}$ mass is observed to have appreciable drop when the magnetic field is increased (but unaffected for $\eta=0.5$ ), when the AMM effects are not taken into account. The $D^{-}$mass has an increasing trend with increase in magnetic field, due to the Landau quantization effects, similar to the $D^{+}$mass. The effects of isospin asymmetry as well as 
AMM effects are observed to be large at higher values of the magnetic fields, and these are more pronounced at high densities. The present study shows that the density effects are the most prominent medium effects and the isospin asymmetry effects as well as AMM effects are appreciable at high densities.

\section{SUMMARY}

We have studied the medium modifications of the open charm mesons, namely the $D$ and $\bar{D}$ mesons, in isospin asymmetric nuclear matter in the presence of strong magnetic fields. These are investigated using a chiral effective model, generalized from SU(3) to $\mathrm{SU}(4)$, to obtain the interactions of these charmed mesons with the light hadrons. The medium modifications of the masses of these mesons in the asymmetric nuclear matter arise due to their interactions with the nucleons and the scalar mesons $(\sigma, \zeta$ and $\delta)$. The number density and scalar density of the proton have contributions from the Landau energy levels in the presence of the magnetic field. The effects of the anomalous magnetic moments of the proton and neutron are also investigated in the present work. The effects of the isospin asymmetry as well as the anomalous magnetic moments of the nucleons are observed to be quite prominent, especially at high densities. The mass modifications of the $D^{+}$and $D^{0}$ mesons of the $D$ meson doublet, as well as of $D^{-}$and $\bar{D}^{0}$ of the $\bar{D}$ doublet, are observed to be quite different in the isospin asymmetric medium at high densities. The effects of

magnetic field on the $D$ and $\bar{D}$ meson masses are observed to be much less as compared to the effects from density, in the present work. The density effects on the in-medium masses of these open mesons are thus the dominant medium effects, which should be observed in the $D^{+} / D^{0}$ and $D^{-} / \overline{D^{0}}$ ratios, as well as in the partial decay widths of the charmonium states to $D^{+} D^{-}$and $D^{0} \bar{D}^{0}$ pairs, in asymmetric heavy ion collisions planned at the Compressed Baryonic Matter (CBM) experiment at FAIR at the future facility at GSI.

\section{Acknowledgments}

The authors thank Hiranmaya Mishra for fruitful discussions. One of the authors (AM) is grateful to the Institut für Theoretische Physik for warm hospitality and acknowledges fi- 
nancial support from Alexander von Humboldt Stiftung when this work was initiated. Amal Jahan CS acknowledges the support towards this work from Department of Science and Technology, Govt of India, via INSPIRE fellowship (Ref. No. DST/INSPIRE/03/2016/003555).

[1] D. Kharzeev, L. McLerran and H. Warringa, Nucl. Phys. A 803, 227 (2008); K. Fukushima, D. E. Kharzeev and H. J. Warringa, Phys. Rev. D 78, 074033 (2008).

[2] V. Skokov, A. Illarionov and V. Toneev, Int. J. Mod. Phys. A 24, 5925 (2009).

[3] C. Thompson and R.C. Duncan, Astrophys. J. 473, 322 (1996); D. Lai and S. L. Shapiro, Astrophys. J. 383, 745 (1991); R.S. Duncan and C. Thompson, Astrophys. J. 392, L9 (1992).

[4] S. Chakrabarty, D. Bandyopadhyay, S. Pal, Phys. Rev. Lett. 78, 2898 (1997).

[5] A. Broderick, M. Prakash and J.M.Lattimer, Astrophys. J. 537, 351 (2002).

[6] A.E. Broderick, M. Prakash and J. M. Lattimer, Phys. Lett. B 531, 167 (2002).

[7] F. X. Wei, G. J. Mao, C. M. Ko, L. S. Kisslinger, H. Stöcker, and W. Greiner, J. Phys. G, Nucl. Part. Phys. 32, 47 (2006).

[8] Guang-Jun Mao, Akira Iwamoto, Zhu-Xia Li, Chin. J. Astrophys. 3, 359 (2003).

[9] Somenath Chakrabarty, Phys. Rev. D 54, 1306 (1996).

[10] Bhaswar Chatterjee, Hiranmaya Mishra and Amruta Mishra, Phys. Rev. D 84, 014016 (2011).

[11] Shovkovy I.A. (2013) Magnetic Catalysis: A Review. In: Kharzeev D., Landsteiner K., Schmitt A., Yee HU. (eds) Strongly Interacting Matter in Magnetic Fields. Lecture Notes in Physics, vol 871. Springer, Berlin, Heidelberg.

[12] K. Tuchin, Phys. Rev. C 83, 017901 (2011); K. Marasinghe and K. Tuchin, Phys. Rev. C 84, 044908 (2011).

[13] K. Tuchin, Phys. Rev. C 83, 034904 (2010), K. Tuchin, Erratum Phys. Rev. C 83, 039903(E) (2011).

[14] Arpan Das, S. S. Dave. P.S. Saumia and A.M. Srivastava, Phys. Rev. C 96, 034902 (2017).

[15] P. V. Buividovich, M. N. Chernodub, D. E. Kharzeev, T. Kalaydhyan, M. I. Polikarpov, Phys. Rev. Lett. 132001 (2010).

[16] K. Tuchin, Phys. Rev. C 88, 024911 (2013). 

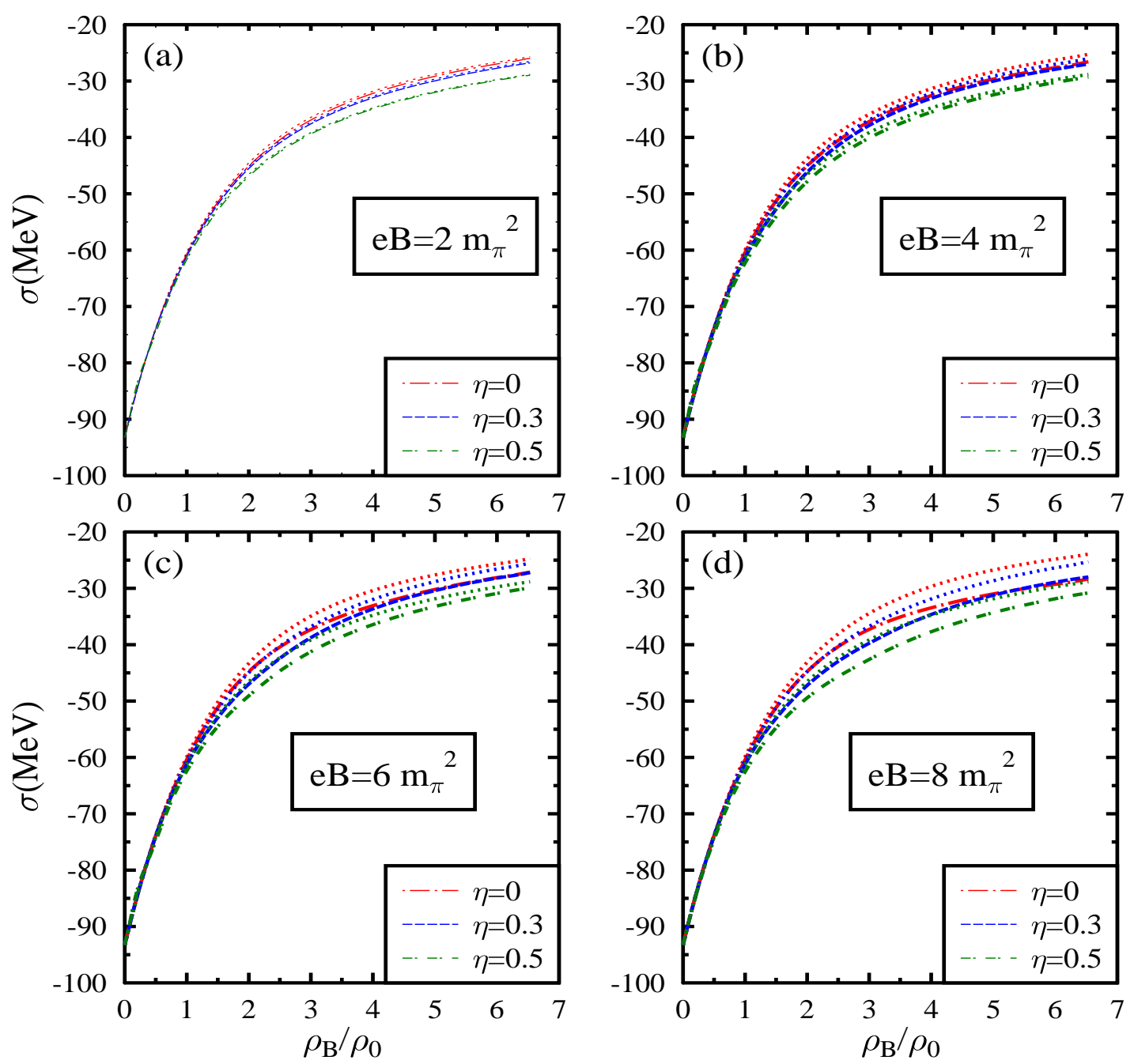

FIG. 1: The scalar field $\sigma$ plotted as function of the baryon density, $\rho_{B} / \rho_{0}$, for different values of magnetic field, is shown for different values of the isospin asymmetry parameter, $\eta$, when the effects of anomalous magnetic moment are taken into account, and are compared to the case when the effects of anomalous magnetic moment is not taken into account (dotted line). 

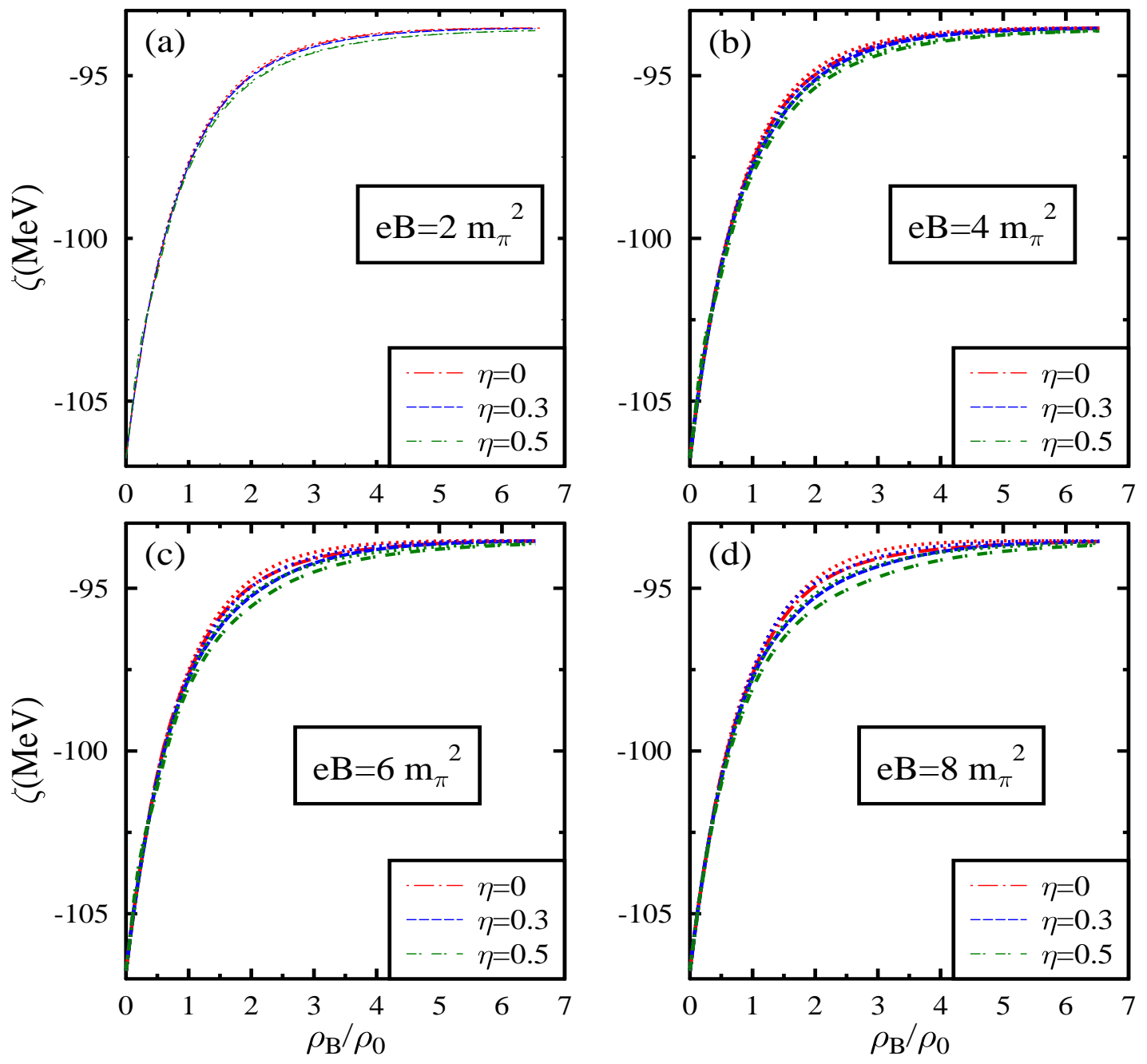

FIG. 2: The scalar field $\zeta$ plotted as function of the baryon density, $\rho_{B} / \rho_{0}$, for different values of magnetic field, is shown for different values of the isospin asymmetry parameter, $\eta$, when the effects of anomalous magnetic moment are taken into account, and are compared to the case when the effects of anomalous magnetic moment is not taken into account (dotted line). 

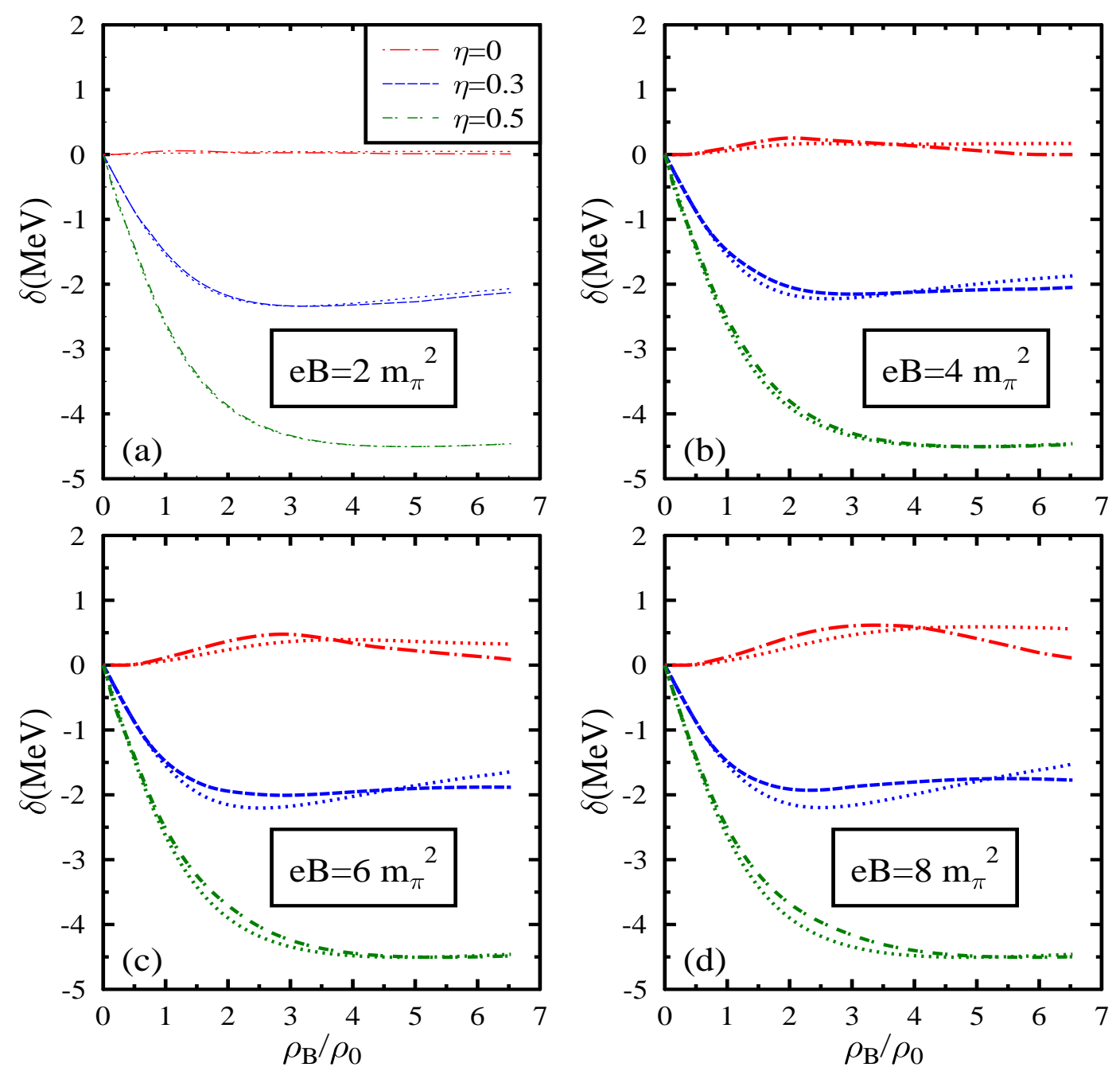

FIG. 3: The scalar field $\delta$ plotted as function of the baryon density, $\rho_{B} / \rho_{0}$, for different values of magnetic field, is shown for different values of the isospin asymmetry parameter, $\eta$, when the effects of anomalous magnetic moment are taken into account, and are compared to the case when the effects of anomalous magnetic moment is not taken into account (dotted line). 

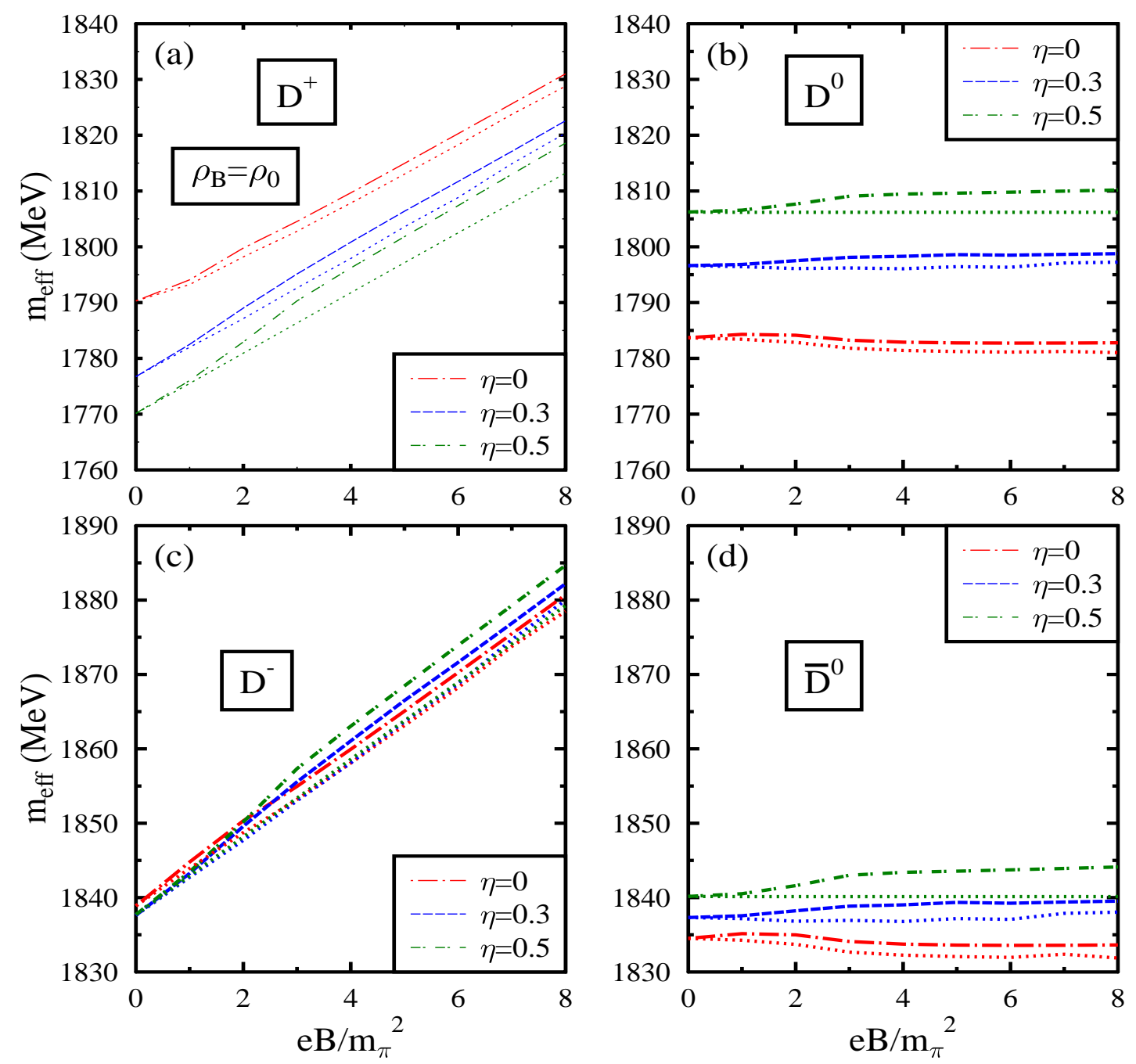

FIG. 4: The effective masses of $D\left(D^{+}, D^{0}\right)$ and $\bar{D}\left(D^{-}, \bar{D}^{0}\right)$ mesons in $\mathrm{MeV}$ plotted as functions of $e B / m_{\pi}^{2}$, for baryon density, $\rho_{B}=\rho_{0}$, with different values of isospin asymmetry parameter, $\eta$, accounting for the effects of the anomalous magnetic moments (AMM) for the nucleons. The results are compared with the case of not accounting for the anomalous magnetic moments (shown as dotted lines). 

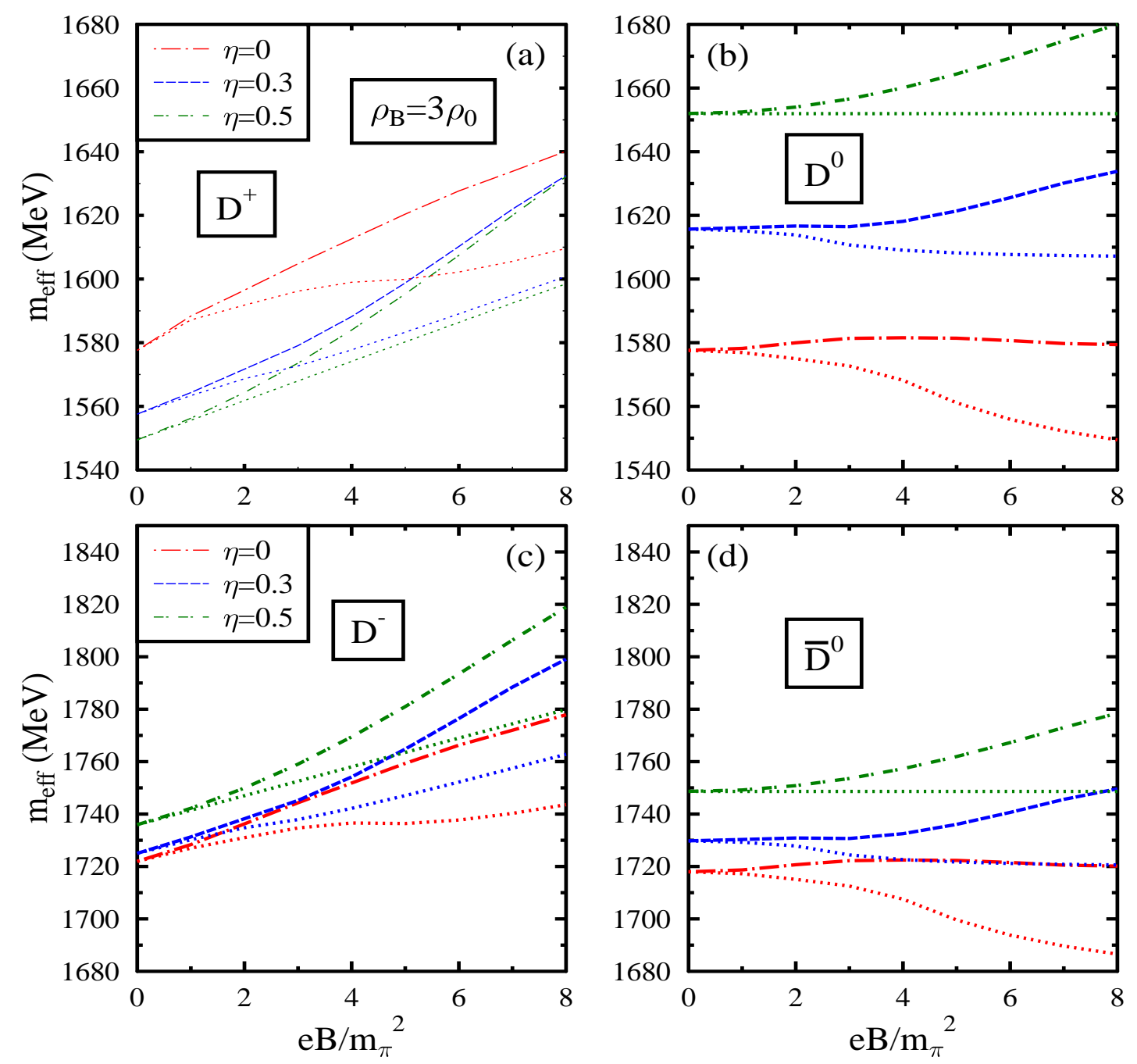

FIG. 5: The effective masses of $D\left(D^{+}, D^{0}\right)$ and $\bar{D}\left(D^{-}, \bar{D}^{0}\right)$ mesons in $\mathrm{MeV}$ plotted as functions of $e B / m_{\pi}^{2}$, for baryon density, $\rho_{B}=3 \rho_{0}$, with different values of isospin asymmetry parameter, $\eta$, accounting for the effects of the anomalous magnetic moments (AMM) for the nucleons. The results are compared with the case of not accounting for the anomalous magnetic moments (shown as dotted lines). 

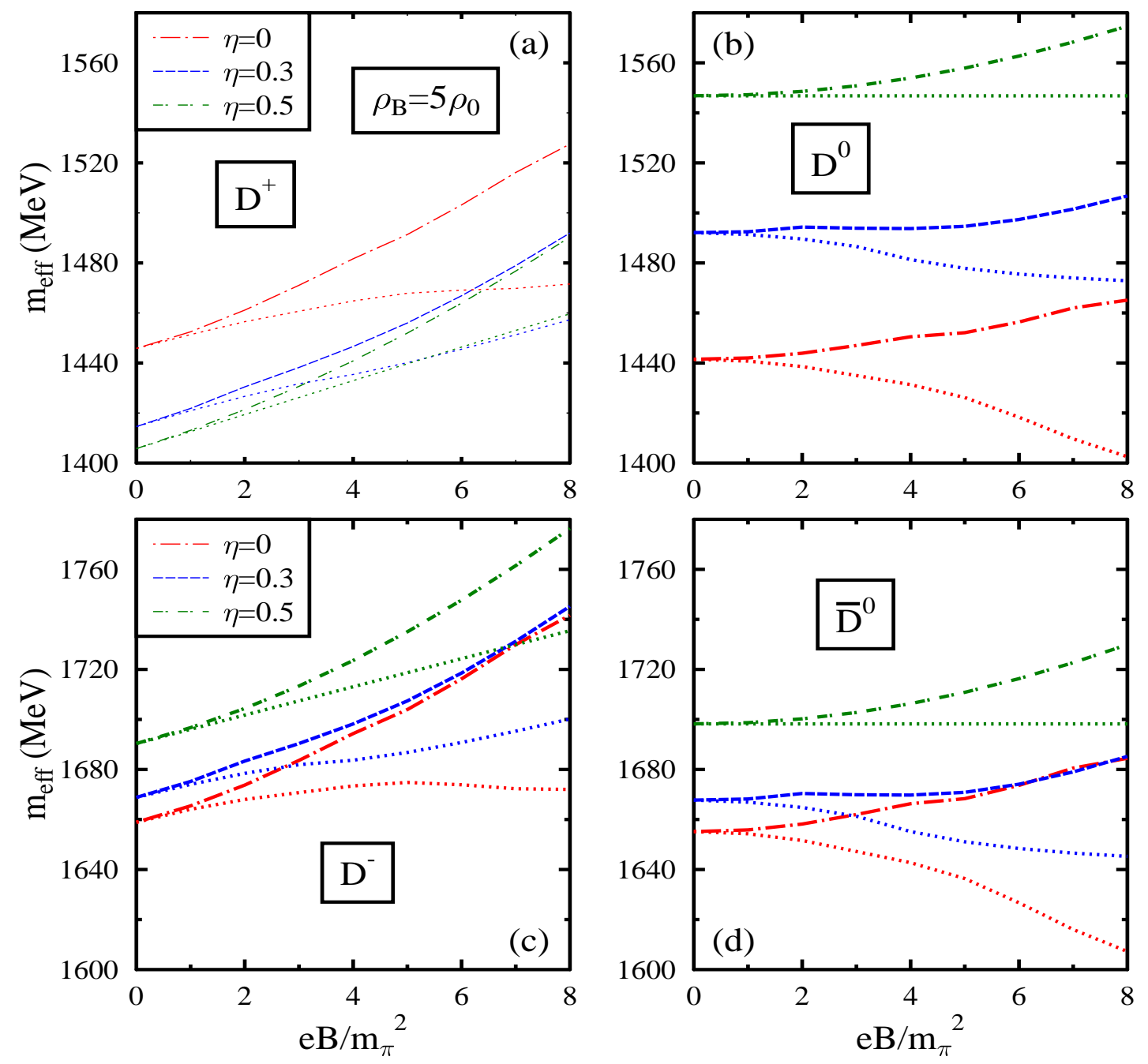

FIG. 6: The effective masses of $D\left(D^{+}, D^{0}\right)$ and $\bar{D}\left(D^{-}, \bar{D}^{0}\right)$ mesons in MeV plotted as functions of $e B / m_{\pi}^{2}$, for baryon density, $\rho_{B}=5 \rho_{0}$, with different values of isospin asymmetry parameter, $\eta$, accounting for the effects of the anomalous magnetic moments (AMM) for the nucleons. The results are compared with the case of not accounting for the anomalous magnetic moments (shown as dotted lines). 
[17] C. S. Machado, F. S. Navarra, E. G. de Oliveira and J. Noronha, Phys. Rev. D 88, 034009 (2013).

[18] C. S. Machado, R. D. Matheus, S. I. Finazzo and J. Noronha, Phys. Rev. D 89, 074027 (2014).

[19] P. Gubler, K. Hattori, S. H. Lee, M. Oka, S. Ozaki and K. Suzuki, Phys. Rev. D 93, 054026 (2016).

[20] S. Cho, K. Hattori, S. H. Lee, K. Morita and S. Ozaki, Phys. Rev. D 91, 045025 (2015).

[21] A. Hayashigaki, Phys. Lett. B 487, 96 (2000).

[22] T. Hilger, R. Thomas, B. Kämpfer, Phys. Rev. C 79, 025202 (2009).

[23] K. Tsushima, D. H. Lu, A. W. Thomas, K. Saito, and R. H. Landau, Phys. Rev. C 59, 2824 (1999); A. Sibirtsev, K. Tsushima, and A. W. Thomas, Eur. Phys. J. A 6, 351 (1999).

[24] P. A. M. Guichon, Phys. Lett. B 200, 235 (1988); K. Saito and A. W. Thomas, Phys. Lett B 327, 9 (1994); K. Saito, K. Tsushima and A. W. Thomas, Nucl. Phys. A 609, 339 (1996); P.K. Panda, A. Mishra, J. M. Eisenberg and W. Greiner, Phys. Rev. C 56, 3134 (1997).

[25] A. Mishra, E. L. Bratkovskaya, J. Schaffner-Bielich, S.Schramm and H. Stöcker, Phys. Rev. C 69, 015202 (2004).

[26] Amruta Mishra and Arindam Mazumdar, Phys. Rev. C 79, 024908 (2009).

[27] Arvind Kumar and Amruta Mishra, Phys. Rev. C 81, 065204 (2010).

[28] Arvind Kumar and Amruta Mishra, Eur. Phys. A 47, 164 (2011).

[29] L.Tolos, J. Schaffner-Bielich and A. Mishra, Phys. Rev. C 70, 025203 (2004).

[30] L. Tolos, J. Schaffner-Bielich and H. Stöcker, Phys. Lett. B 635, 85 (2006).

[31] T. Mizutani and A. Ramos, Phys. Rev. C 74, 065201 (2006); L.Tolos, A. Ramos and T. Mizutani, Phys. Rev. C 77, 015207 (2008).

[32] J. Hofmann and M.F.M.Lutz, Nucl. Phys. A 763, 90 (2005).

[33] Z-G. Wang and Tao Huang, Phys. Rev. C 84, 048201 (2011).

[34] Z-G. Wang, Phys. Rev. C 92, 065205 (2015).

[35] T. Hilger and B. Kaempfer, Nucl. Phys. B (Proc. Suppl.) 207-208, 277 (2010).

[36] T. Hilger and B. Kampfer and S. Leupold, Phys. Rev. C 84, 045202 (2011).

[37] Rahul Chhabra and Arvind Kumar, Eur. Phys. J A 53, 105 (2017); ibid, Eur. Phys. J C 77, 726 (2017), Arvind Kumar and Rahul Chhabra, Phys. Rev. C 92, 035208 (2015). 
[38] R. Molina, D. Gamermann, E. Oset, and L. Tolos, Eur. Phys. J A 42, 31 (2009); L. Tolos, R. Molina, D. Gamermann, and E. Oset, Nucl. Phys. A 827 249c (2009).

[39] P. Papazoglou, D. Zschiesche, S. Schramm, J. Schaffner-Bielich, H. Stöcker, and W. Greiner, Phys. Rev. C 59, 411 (1999).

[40] A. Mishra, K. Balazs, D. Zschiesche, S. Schramm, H. Stöcker, and W. Greiner, Phys. Rev. C 69, $024903(2004)$.

[41] D. Zschiesche, A. Mishra, S. Schramm, H. Stöcker and W. Greiner, Phys. Rev. C 70, 045202 (2004).

[42] A. Mishra, E. L. Bratkovskaya, J. Schaffner-Bielich, S. Schramm, H. Stöcker, Phys. Rev. C 70, $044904(2004)$.

[43] A. Mishra and S. Schramm, Phys. Rev. C 74, 064904 (2006).

[44] A. Mishra, S. Schramm and W. Greiner, Phys. Rev. C 78, 024901 (2008).

[45] Amruta Mishra, Arvind Kumar, Sambuddha Sanyal, S. Schramm, Eur. Phys. J A 41, 205 (2009).

[46] Amruta Mishra, Arvind Kumar, Sambuddha Sanyal, V. Dexheimer, S. Schramm, Eur. Phys. J 45, 169 (2010).

[47] Divakar Pathak and Amruta Mishra, Adv. High Energy Phys. 2015, 697514 (2015).

[48] J. Schechter, Phys. Rev. D 21, 3393 (1980).

[49] Amruta Mishra and Divakar Pathak, Phys. Rev. C 90, 025201 (2014).

[50] B. Friman, S. H. Lee and T. Song, Phys. Lett, B 548, 153 (2002).

[51] A. Le Yaouanc, L. Oliver, O. Pene and J. C. Raynal, Phys. Rev. D 8, 2223 (1973); ibid, Phys. Rev. D 9, 1415 (1974); ibid, Phys. Rev. D 11, 1272 (1975).

[52] T. Barnes, F. E. Close, P. R. Page and E. S. Swanson, Phys. Rev. D 55, 4157 (1997).

[53] Amruta Mishra, S. P. Misra and W. Greiner, Int. J. Mod. Phys. E 24, 155053 (2015).

[54] Divakar Pathak and Amruta Mishra, Phys. Rev. C 91, 045206 (2015).

[55] Divakar Pathak and Amruta Mishra, Int. J. Mod. Phy. E 23, 1450073 (2014).

[56] S. Yasui and K. Sudoh, Phys. Rev. C 87, 015202 (2013).

[57] S. H. Lee and C. M. Ko, Phys. Rev. C 67, 038202 (2003).

[58] G. Krein, A. W. Thomas and K. Tsushima, Phys. Lett. B 697, 136 (2011). 
[59] G. Krein, A. W. Thomas and K. Tsushima, arXiv: 1706.02688 (hep-ph).

[60] Amruta Mishra and S. P. Misra, Phy. Rev. C 95, 065206 (2017).

[61] Amruta Mishra, Phys. Rev. C 91, 035201 (2015).

[62] Arvind Kumar and Amruta Mishra, Phys. Rev. C 28, 045207 (2010).

[63] M. Pitschmann and A. N. Ivanov, arXive : 1205.5501 (math-ph).

[64] V. Dexheimer, R. Negreiros, S. Schramm, Eur. Phys. Journal A 48, 189 (2012); V. Dexheimer, B. Franzon and S. Schramm, Jour. Phys. Conf. Ser. 861, 012012 (2017).

[65] R. M. Aguirre and A. L. De Paoli, Eur. Phys. J. A 52, 343 (2016).

[66] R. M. Aguirre, Phys. Rev. D 96, 096013 (2017).

[67] S.Weinberg, Phys. Rev. 1661568 (1968).

[68] S. Coleman, J. Wess, B. Zumino, Phys. Rev. 1772239 (1969); C.G. Callan, S. Coleman, J. Wess, B. Zumino, Phys. Rev. 1772247 (1969).

[69] W. A. Bardeen and B. W. Lee, Phys. Rev. 1772389 (1969).

[70] G. E. Brown, C-H Lee, M. Rho, and V. Thorsson, Nucl. Phys. A567, 937 (1994).

[71] J. Schaffner-Bielich, I. N. Mishustin, J. Bondorf, Nucl. Phys. A 625, 325 (1997).

[72] T. Barnes and E. S. Swanson, Phys. Rev. C 49, 1166 (1994). 